\title{
Simultaneous degradation of nitrogenous heterocyclic compounds by catalytic wet-peroxidation process using box-behnken design
}

\author{
Vijayalakshmi Gosu ${ }^{1}$, Shivali Arora ${ }^{2}$, Verraboina Subbaramaiah ${ }^{2+}$ \\ ${ }^{1}$ Department of Chemical Engineering, MBM Engineering College, Jodhpur 342011, India \\ ${ }^{2}$ Department of Chemical Engineering, MNIT Jaipur, Jaipur 302017, India
}

\begin{abstract}
The present study investigates the feasibility of nitrogenous heterocyclic compounds (NHCs) (Pyridine-Quinoline) degradation by catalytic wet peroxidation (CWPO) in the presence of nanoscale zerovalent iron supported on granular activated carbon (nFe $/ \mathrm{GAC}$ ) using statistical optimization technique. Response surface methodology (RSM) in combination with Box-Behnken design (BBD) was used to optimize the process parameters of CWPO process such as initial $\mathrm{pH}$, catalyst dose, hydrogen peroxide dose, initial concentration of pyridine (Py) and quinolone (Qn) were chosen as the main variables, and total organic carbon (TOC) removal and total Fe leaching were selected as the investigated response. The optimization of process parameters by desirability function showed the $\sim 85 \%$ of TOC removal with process condition of initial solution $\mathrm{pH}$ 3.5 , catalyst dose of $0.55 \mathrm{~g} / \mathrm{L}$, hydrogen peroxide concentration of $0.34 \mathrm{mmol}$, initial concentration of Py $200 \mathrm{mg} / \mathrm{L}$ and initial concentration of Qn $200 \mathrm{mg} / \mathrm{L}$. Further, for TOC removal the analysis of variance results of the RSM revealed that all parameter i.e. initial pH, catalyst dose, hydrogen peroxide dose, initial concentration of Py and initial concentration of Qn were highly significant according to the $p$ values $(p<0.05)$. The quadratic model was found to be the best fit for experimental data. The present study revealed that BBD was reliable and effective for the determination of the optimum conditions for CWPO of NHCs (Py-Qn).
\end{abstract}

Keywords: Box-Benken Design, Catalytic wet peroxidation, CWPO, Nitrogenous heterocyclic compounds, Response surface methodology

\section{Introduction}

Nitrogenous heterocyclic compounds (NHCs) releases into a water/ wastewater stream due to its wide applicability in chemical industries such as coal tar, dye, fertilizer, insecticides, and pharmaceuticals [1]. Because of nitrogen-atom included in the ring system, these compounds have high water solubility. NHCs have been listed as hazardous and toxic chemical due to its adverse impact on the natural environment. These compounds are toxic, potentially carcinogenic and mutagenic properties even at low concentration of NHCs in the aqueous stream [2]. Among these compounds, Py and Qn selected as a model compounds under NHCs category. Furthermore, the Py has listed as a priority of organic pollutant by the United States Environmental Protection Agency (USEPA). It was reported that shale oil process water contains Py and Qn in the range of $20 \pm 100 \mathrm{mg} / \mathrm{L}$ [3].

Numerous studies have been reported for the treatment of NHCs by biological processes such as aerobic and anaerobic process.
Limited success was achieved by the biological process because it requires long residence time to degrade NHCs [4, 5]. Moreover, NHCs are hard to remove and are toxic to microbial communities. For medium strength wastewater, the thermal treatment process (incineration) is not a feasible option because of the high energy requirement [6].

Therefore, an advanced oxidation process is one of the alternative for the treatment of refractory organic compounds [7]. Among various advanced oxidation process, catalytic wet peroxidation (CWPO) received rapid attention because it may non-selectively degrade the non-biodegradable compounds into harmless or simple compounds in the presence of oxidant using homogeneous/heterogeneous catalyst under atmospheric pressure and moderate temperature (below $80^{\circ} \mathrm{C}$ ) [8-10]. In recent years many researchers are focused on the development of a novel heterogeneous catalyst for CWPO process. Nanoscale iron particles $\left(\mathrm{nFe}^{0}\right)$ stand for a new generation of environmental remediation technologies [11]. This $\mathrm{nFe}^{0}$ possess distinctive chemical, electronic, magnetic, mechan-
This is an Open Access article distributed under the terms of the Creative Commons Attribution Non-Commercial License (http://creativecommons.org/licenses/by-nc/3.0/) which permits unrestricted non-commercial use, distribution, and reproduction in any medium, provided the original work is properly cited.

Copyright (C) 2020 Korean Society of Environmental Engineers
Received April 4, 2019 Accepted July 6, 2019

${ }^{\dagger}$ Corresponding author

Email: vsr.chem@mnit.ac.in

Tel: +91-8560073299

ORCID: 0000-0003-4457-6725 
ical, optical and catalytic properties have drawn immense attention on zero-valent iron [12]. And also, provide cost-effective solutions to most exigent environmental cleanup problems. Nowadays, most of the scientists are paying attention to dispersibility of $\mathrm{nFe}^{0}$ on various support materials, particularly on porous materials in order increase the available surface area for reaction, enhance the stability, and minimize the agglomeration and leaching $[13,14]$. In the present study nanoscale zerovalent iron supported on granular activated carbon ( $\mathrm{nFe}^{0} / \mathrm{GAC}$ or $\left.\mathrm{nZVI} / \mathrm{GAC}\right)$ was synthesized and subjected to CWPO of NHCs.

In recent years, researchers are focused on the statistical experimental approach. It is a very useful technique for the optimization of any process conditions using mathematical models and also to determine the significant parameters and their interaction with other parameters by conducting the minimum number of experiments. Traditional optimization methods are a time-consuming process; they can evaluate only one factor at a time. Further, it requires a large number of experimental runs to find out the optimum level [15]. Box-Behnken design (BBD) is one of the statistical optimization tools for analysis of multiple variables, with a limited number of experiments [16]. The merits of BBD over other design methods are it avoids the extreme conditions that can skew the outcome. Therefore, all factors are simultaneously tested at their lowest levels to the highest level.

The objective of this study is to investigate the significant operating parameters for the simultaneous degradation of NHCs (Py-Qn) by CWPO process using BBD. The design consists of five factors and three levels of each factor, which are used to optimize the TOC removal by $\mathrm{nFe}^{0} / \mathrm{GAC}$.

\section{Materials and Methods}

\subsection{Material}

All chemicals used in these studies were of analytical reagent (AR) grade without any additional purification. Quinoline, pyridine, ferrous sulfate heptahydrate, ethanol, $\mathrm{NaOH}$ and $\mathrm{H}_{2} \mathrm{SO}_{4}$, were obtained from SD fine chemicals, India. Hydroxylamine hydrochloride, sodium borohydride, 1,10-phenanthroline were purchased from Sigma Aldrich. 30-33\% hydrochloric acid, dichloromethane, acetic acid and 30\% hydrogen peroxide were procured from Rankem, India. All analytical standards were prepared from Millipore water.

\subsection{Synthesis of $20 \% n \mathrm{nFe} / \mathrm{GAC}$}

Sodium borohydride method was used for the synthesis of nanoscale zero-valent iron incorporated on granular activated carbon $(20 \%$ $\mathrm{nFe}^{0} / \mathrm{GAC}$ ) [14]. The commercial grade GAC was washed with distilled water to remove impurities and soaked in hot distilled water at $100^{\circ} \mathrm{C}$ to increase the activation of pores. Soaked activated carbon filtered and dried in an oven at $120^{\circ} \mathrm{C}$ for overnight. $9.956 \mathrm{~g}$ of $\mathrm{FeSO}_{4} .7 \mathrm{H}_{2} \mathrm{O}$ was used as an iron precursor mixed with ethanol and water solution $(80 \mathrm{~mL}$ ethanol $+20 \mathrm{~mL}$ water) with continuous stirring for $10 \mathrm{~min}$. After complete dissolution of the iron precursor in the solution, $8 \mathrm{~g}$ of prepared GAC was added to iron precursor solution, in order to disperse the ion species uniformly over the
GAC pores, the solution was kept for $20 \mathrm{~min}$ in an ultrasonic shaker. Gosu et al. [18] reported the iron reduction by borohydride is followed.

$$
\begin{aligned}
& \mathrm{Fe}_{a q}^{2+} \mathrm{OrFe}_{s}^{2+} / \mathrm{GAC}+\mathrm{BH}_{4 a q}^{-}+6 \mathrm{H}_{2} \mathrm{O}_{(I)(a q)} \rightarrow \\
& \mathrm{Fe}_{s}^{0}+\mathrm{Fe}_{s}^{0} / \mathrm{GAC} \downarrow+2 \mathrm{~B}(\mathrm{OH})_{3(a q)}+7 \mathrm{H}_{2(g)} \uparrow
\end{aligned}
$$

In the above equation, Eq. (1), $\mathrm{Fe}_{s}^{2+} / G A C$ indicates iron species incorporated in the GAC framework and $\mathrm{Fe}_{s}^{0} / G A C$ indicates as nanoscale zero-valent iron species incorporated in the GAC framework.

The strong reducing agent $\mathrm{NaBH}_{4}$ was dissolved in $100 \mathrm{~mL}$ ultra-pure deionized water based on stoichiometric quantity and further added into the sonicated solution with continuous stirring. The black solid particles immediately appeared during the dropwise addition of the reducing agent, which indicates the formation of $\mathrm{Fe}^{0}$ on granular activated carbon. The complete solution filtered by using a vacuum filtration unit and the filtrate washed with ethanol for two to three times to remove water. At $50^{\circ} \mathrm{C}$, the synthesized $\mathrm{nFe} / \mathrm{GAC}$ were dried and designated as $20 \% \mathrm{nFe}^{0} / \mathrm{GAC}$ or nZVI/GAC

\subsection{Batch Experimental Program}

The experiments were carried out in a three-necked round bottom glass reactor equipped with a reflux system to condense the vapor samples and to minimize the experimental error. The reactor was kept inside the oil bath to maintain the uniform desired temperature. The whole setup was mounted on a magnetic stirrer with a hot plate (2MLH, REMI). The temperature of the reaction mixture was increased using a proportional-integral-derivative controller. The reaction mixture was uniformly maintained with a magnetic stirrer at $300 \mathrm{rpm}$. For each experimental run, a $100 \mathrm{~mL}$ reaction mixture initial $\mathrm{pH}$ was adjusted (2-10 $\mathrm{pH}$ ) by using $0.1 \mathrm{~N} \mathrm{HCl}$ and 0.1 $\mathrm{N} \mathrm{NaOH}$ solution and then charged into the reactor. After that, the reaction mixture heated to $60^{\circ} \mathrm{C}$, with the help of oil bath, and required quantities of catalyst dose ( $\left.\mathrm{nFe}^{0} / \mathrm{GAC}\right)$ and oxidant (hydrogen peroxide) were added to the reaction mixture. During the reaction, $\mathrm{pH}$ was not controlled. After completion of reaction $5 \mathrm{~h}$, the sample in the reactor was filtered using $0.45 \mu \mathrm{m}$ syringe membrane filter (PTFE-2545, MOXCARE), and the filtrate samples were analyzed with a TOC analyzer.

\subsection{Analytical Methods}

Mineralization of pyridine and quinoline was quantified with the help of total organic carbon (TOC) conversion. TOC was quantified by catalytic oxidation of organic compounds into $\mathrm{CO}_{2}$, the formed $\mathrm{CO}_{2}$ quantified using non-dispersive infrared (NDIR) detector by TOC-VCPH-analyzer (Shimadzu 5500A, Germany). The instrument measure the total carbon by combustion of the sample at $700^{\circ} \mathrm{C}$ over a Pt catalyst bed and total inorganic carbon was measured by treating the sample with $25 \%$ phosphoric acid. TOC obtained by subtracting total inorganic carbon from total carbon. TOC values present the average of at least two measurements, in some cases samples were measured three times by injecting three times which is evaluated with the TOC apparatus. For the quantification of 
Fe in the aqueous solution, all the experimental samples were digested and further iron reduced to the ferrous state using hydroxylamine hydrochloride. The 1,10-phenanthroline was used as a ligand that reacts with metal (Fe) to form a strongly coloured complex. The coloured complex was measured at intensity $510 \mathrm{~nm}$ with the help of UV visible spectrophotometer (HACH, DR 5000, USA) [17]. Each sample was measured triplicate and results are presented with $\pm 5 \%$ deviation from the average value.

\subsection{Catalyst Characterization}

Micromeritics ASAP 2020 instrument was used to determine the surface area and pore volume with the help of $\mathrm{N}^{2}$-adsorption and desorption isotherm. Samples were pretreated by degassing the samples at $150^{\circ} \mathrm{C}$ under the vacuum of $10-3$ torr for $6 \mathrm{~h}$. Micropore volume was calculated using the t-plot and surface area was calculated using the Brunauer-Emmett-Teller (BET) equation by assuming that all pores in the sample are cylindrical and parallel.

Scanning electron microscopy (SEM-501 Phillips, Holland) employed to analyze the surface morphology of synthesized catalyst by operating at acceleration voltage $15-25 \mathrm{kV}$ and magnification values up to 40000X. Before SEM analysis, samples were pretreated by gold coating with sputter coater thereby enhances the conductivity of the samples.

\subsection{Box-Behnken Design}

In this study, three-level and five-factorial BBD design were employed to optimize the process variables resulting in maximum TOC removal and minimum Iron leaching. BBD estimates the critical operating condition by second-order multivariate. BBD design consist of variable combinations at the center and at middle points of the edges by rotatable quadratic design [15].

The design composed of 3 levels which were coded as -1 (low), 0 (central point or middle) and 1 (high). The process variables are initial $\mathrm{pH}(\mathrm{A})$, hydrogen peroxide dose (B), catalyst dose (C), Py concentration $\left(\mathrm{C}_{\mathrm{Py}}\right)(\mathrm{D})$ and $\mathrm{Qn}$ concentration $\left(\mathrm{C}_{\mathrm{Qn}}\right)(\mathrm{E})$ shown in Table 1. The ranges of the process variables are selected based on the preliminary experiments. The statistical analysis was performed using Design-Expert ${ }^{\mathrm{TM}}$ software. The corresponding design matrix consists of 46 experiments with center point. The experimental design matrix by the BBD is tabulated in Table 2 . To fit the mathematic model, analysis of variance (ANOVA) and multiple regression analysis were estimated. The Experimental data was analyzed with multiple regressions in order to fit the data in the second-order polynomial equation (Eq. (2)). It can be written as

$$
\begin{aligned}
Y= & \beta_{0}+\beta_{1} A+\beta_{2} B+\beta_{3} C+\beta_{4} D+\beta_{5} E+\beta_{11} A^{2}+ \\
& \beta_{22} B^{2}+\beta_{33} C^{2}+\beta_{44} D^{2}+\beta_{55} E^{2}+ \\
& \beta_{12} A B+\beta_{13} A C+\beta_{14} A D+\beta_{15} A E+\beta_{23} B C+ \\
& \beta_{24} B D+\beta_{25} B E+\beta_{34} C D+\beta_{35} C E+\varepsilon
\end{aligned}
$$

Where $\mathrm{Y}$ is the response and $\mathrm{A}, \mathrm{B}, \mathrm{C}, \mathrm{D}$ and $\mathrm{E}$ are process variables. $A^{2}, B^{2}, C^{2}, D^{2}$ and $E^{2}$ are square of process variables, $\mathrm{AB}, \mathrm{AC}, \mathrm{AD}, \mathrm{AE}, \mathrm{BC}, \mathrm{BD}, \mathrm{BE}, \mathrm{CD}$ and $\mathrm{CE}$ are interaction effect of process variables. $\beta_{0}$ and $\varepsilon$ are constants. $\beta_{1}, \beta_{2}, \beta_{3}, \beta_{4}$ and $\beta_{5}$ are linear coefficients $\beta_{12}, \beta_{13}, \beta_{14}, \beta_{15}, \beta_{23}, \beta_{24}, \beta_{25}, \beta_{34}$ and $\beta_{35}$ are interaction coefficients.

\section{Result and Discussion}

\subsection{Catalyst Characterization}

Surface area and textural properties of GAC and $20 \% \mathrm{nFe} / \mathrm{GAC}$ was studied by $\mathrm{N}_{2}$ adsorption-desorption isotherms. Shape and behavior of the nitrogen adsorption-desorption isotherms of GAC and $20 \% \mathrm{nFe}^{0} / \mathrm{GAC}$ are illustrated in Fig. 1. According to IUPAC classification, the GAC and $20 \% \mathrm{nFe}^{0} / \mathrm{GAC}$ exhibits the type IV hysteresis based on nitrogen adsorption-desorption isotherms with the relative pressures $\left(\mathrm{p} / \mathrm{p}_{0}\right)$ in the range of 0.2 to 0.8 that may be attributed to the characteristic of mesoporous structure. In addition, the observations from the nitrogen adsorption-desorption loop

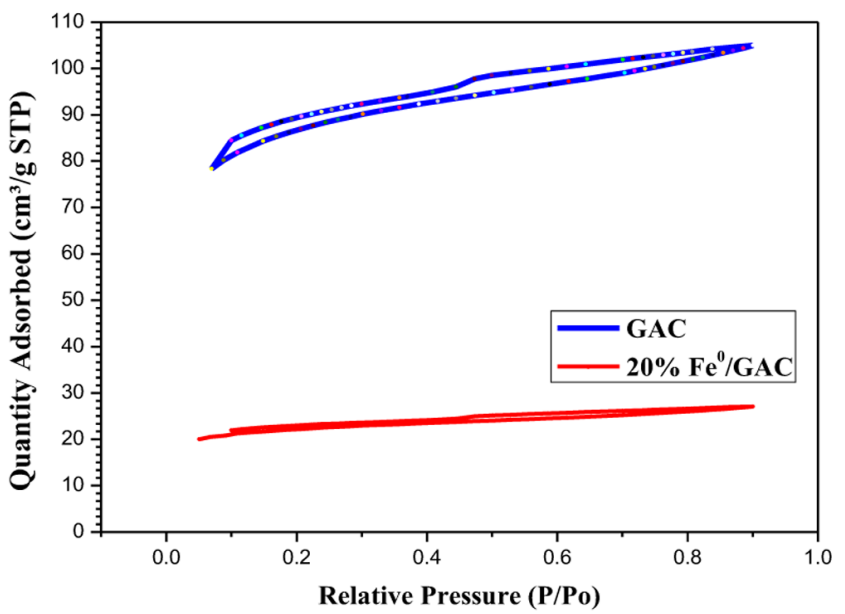

Fig. 1. $\mathrm{N}_{2}$ - adsorption - desorption isotherms on $\mathrm{CAC}$ and $20 \% \mathrm{nFe} / \mathrm{GAC}$.

Table 1. Process Variables and Experimental Box-Behnken Design Levels

\begin{tabular}{lcccc}
\hline \multirow{2}{*}{ Process Variables } & Factors & \multicolumn{2}{c}{ Levels } \\
\cline { 2 - 5 } \cline { 2 - 2 } & X & Low(-1) & Middle(0) & High(+1) \\
Hydrogen peroxide dose (mmol) & A & 2 & 0.33 & 6 \\
Catalyst dose, m (g/L) & B & 0.15 & 0.55 & 1 \\
Pyridine con. (C) $(\mathrm{mg} / \mathrm{L})$ & $\mathrm{C}$ & 0.1 & 100 & 200 \\
Quinoline con. $\left(\mathrm{C}_{\mathrm{Qn}}\right)(\mathrm{mg} / \mathrm{L})$ & $\mathrm{D}$ & 0 & 100 & 200 \\
\hline
\end{tabular}


Table 2. Box - Behnken Design Matrix and the Responses of the Dependent Factors and Predicted Values

\begin{tabular}{|c|c|c|c|c|c|c|c|c|c|c|}
\hline \multirow{2}{*}{$\begin{array}{l}\text { Std } \\
\text { Order }\end{array}$} & \multirow{2}{*}{$\begin{array}{l}\text { Run } \\
\text { Order }\end{array}$} & \multirow{2}{*}{$\mathbf{p H}$} & \multirow{2}{*}{$\begin{array}{c}\mathrm{H}_{2} \mathrm{O}_{2} \\
\text { (mmol) }\end{array}$} & \multirow{2}{*}{$\underset{(g / L)}{m}$} & \multirow{2}{*}{$\begin{array}{c}\mathrm{C}_{\mathrm{Py}} \\
(\mathrm{mg} / \mathrm{L})\end{array}$} & \multirow{2}{*}{$\begin{array}{c}\mathrm{C}_{\mathrm{Qn}} \\
(\mathrm{mg} / \mathrm{L})\end{array}$} & \multicolumn{2}{|c|}{ \% Removal of TOC } & \multicolumn{2}{|c|}{ Total Fe leaching (mg/L) } \\
\hline & & & & & & & $\mathbf{Y}_{\exp }$ & $\mathbf{Y}_{\text {pred }}$ & $\mathbf{Y}_{\exp }$ & $\mathbf{Y}_{\text {pred }}$ \\
\hline 1 & 25 & 2 & 0.15 & 0.55 & 100 & 100 & 59.98 & 59.98 & 0.45 & 0.46 \\
\hline 2 & 14 & 6 & 0.15 & 0.55 & 100 & 100 & 31.74 & 31.74 & 0.92 & 0.97 \\
\hline 3 & 32 & 2 & 0.5 & 0.55 & 100 & 100 & 60.21 & 60.21 & 1.19 & 1.21 \\
\hline 4 & 39 & 6 & 0.5 & 0.55 & 100 & 100 & 55.64 & 55.64 & 0.01 & 0.05 \\
\hline 5 & 30 & 4 & 0.325 & 0.1 & 0 & 100 & 58.93 & 58.93 & 0.22 & 0.20 \\
\hline 6 & 33 & 4 & 0.325 & 1 & 0 & 100 & 82.80 & 82.80 & 0.80 & 0.77 \\
\hline 7 & 3 & 4 & 0.325 & 0.1 & 200 & 100 & 57.22 & 57.22 & 0.33 & 0.33 \\
\hline 8 & 41 & 4 & 0.325 & 1 & 200 & 100 & 62.23 & 62.23 & 0.60 & 0.60 \\
\hline 9 & 11 & 4 & 0.15 & 0.55 & 100 & 0 & 80.14 & 80.14 & 0.54 & 0.55 \\
\hline 10 & 1 & 4 & 0.5 & 0.55 & 100 & 0 & 67.01 & 67.01 & 0.69 & 0.70 \\
\hline 11 & 18 & 4 & 0.15 & 0.55 & 100 & 200 & 40.90 & 40.90 & 1.11 & 1.13 \\
\hline 12 & 43 & 4 & 0.5 & 0.55 & 100 & 200 & 78.16 & 78.16 & 0.78 & 0.80 \\
\hline 13 & 27 & 2 & 0.325 & 0.1 & 100 & 100 & 50.72 & 50.72 & 0.75 & 0.77 \\
\hline 14 & 40 & 6 & 0.325 & 0.1 & 100 & 100 & 31.63 & 31.63 & 0.17 & 0.21 \\
\hline 15 & 9 & 2 & 0.325 & 1 & 100 & 100 & 62.47 & 62.47 & 0.94 & 0.96 \\
\hline 16 & 37 & 6 & 0.325 & 1 & 100 & 100 & 48.76 & 48.76 & 0.82 & 0.87 \\
\hline 17 & 19 & 4 & 0.325 & 0.55 & 0 & 0 & 98.12 & 98.12 & 0.23 & 0.24 \\
\hline 18 & 45 & 4 & 0.325 & 0.55 & 200 & 0 & 65.49 & 65.49 & 0.57 & 0.61 \\
\hline 19 & 46 & 4 & 0.325 & 0.55 & 0 & 200 & 62.58 & 62.58 & 0.95 & 0.97 \\
\hline 20 & 23 & 4 & 0.325 & 0.55 & 200 & 200 & 72.93 & 72.93 & 0.52 & 0.56 \\
\hline 21 & 24 & 4 & 0.15 & 0.1 & 100 & 100 & 44.76 & 44.76 & 0.49 & 0.46 \\
\hline 22 & 4 & 4 & 0.5 & 0.1 & 100 & 100 & 54.94 & 54.94 & 0.50 & 0.47 \\
\hline 23 & 35 & 4 & 0.15 & 1 & 100 & 100 & 57.31 & 57.31 & 1.01 & 0.98 \\
\hline 24 & 28 & 4 & 0.5 & 1 & 100 & 100 & 71.26 & 71.26 & 0.82 & 0.79 \\
\hline 25 & 5 & 2 & 0.325 & 0.55 & 0 & 100 & 80.25 & 80.25 & 0.30 & 0.37 \\
\hline 26 & 38 & 6 & 0.325 & 0.55 & 0 & 100 & 51.13 & 51.13 & 0.60 & 0.60 \\
\hline 27 & 12 & 2 & 0.325 & 0.55 & 200 & 100 & 56.39 & 56.39 & 0.91 & 0.90 \\
\hline 28 & 10 & 6 & 0.325 & 0.55 & 200 & 100 & 52.71 & 52.71 & 0.10 & 0.02 \\
\hline 29 & 16 & 4 & 0.325 & 0.1 & 100 & 0 & 62.53 & 62.53 & 0.09 & 0.10 \\
\hline 30 & 44 & 4 & 0.325 & 1 & 100 & 0 & 77.63 & 77.63 & 1.20 & 1.21 \\
\hline 31 & 7 & 4 & 0.325 & 0.1 & 100 & 200 & 49.15 & 49.15 & 1.11 & 1.13 \\
\hline 32 & 17 & 4 & 0.325 & 1 & 100 & 200 & 62.91 & 62.91 & 0.84 & 0.86 \\
\hline 33 & 13 & 2 & 0.325 & 0.55 & 100 & 0 & 78.87 & 78.87 & 0.12 & 0.07 \\
\hline 34 & 26 & 6 & 0.325 & 0.55 & 100 & 0 & 50.94 & 50.94 & 1.29 & 1.24 \\
\hline 35 & 15 & 2 & 0.325 & 0.55 & 100 & 200 & 53.29 & 53.29 & 1.97 & 1.91 \\
\hline 36 & 36 & 6 & 0.325 & 0.55 & 100 & 200 & 48.42 & 48.42 & 0.14 & 0.08 \\
\hline 37 & 31 & 4 & 0.15 & 0.55 & 0 & 100 & 72.25 & 72.25 & 0.49 & 0.47 \\
\hline 38 & 42 & 4 & 0.5 & 0.55 & 0 & 100 & 76.47 & 76.47 & 0.47 & 0.45 \\
\hline 39 & 29 & 4 & 0.15 & 0.55 & 200 & 100 & 53.27 & 53.27 & 0.51 & 0.51 \\
\hline 40 & 2 & 4 & 0.5 & 0.55 & 200 & 100 & 73.18 & 73.18 & 0.36 & 0.36 \\
\hline 41 & 22 & 4 & 0.325 & 0.55 & 100 & 100 & 55.58 & 56.50 & 1.30 & 1.17 \\
\hline 42 & 8 & 4 & 0.325 & 0.55 & 100 & 100 & 55.74 & 56.50 & 1.10 & 1.17 \\
\hline 43 & 20 & 4 & 0.325 & 0.55 & 100 & 100 & 58.36 & 56.50 & 1.32 & 1.17 \\
\hline 44 & 6 & 4 & 0.325 & 0.55 & 100 & 100 & 56.59 & 56.50 & 1.03 & 1.17 \\
\hline 45 & 21 & 4 & 0.325 & 0.55 & 100 & 100 & 57.16 & 56.50 & 1.04 & 1.17 \\
\hline 46 & 34 & 4 & 0.325 & 0.55 & 100 & 100 & 55.58 & 56.50 & 1.23 & 1.17 \\
\hline
\end{tabular}


Table 3. Textural Characteristics of the $\mathrm{nFe} / \mathrm{GAC}$

\begin{tabular}{|c|c|c|c|c|c|}
\hline \multirow{2}{*}{ Sample } & \multicolumn{2}{|c|}{ wt\% } & \multirow{2}{*}{$S^{\mathrm{BET}}\left(\mathrm{m}^{2} / \mathrm{g}\right)$} & \multirow{2}{*}{$V_{p}\left(\mathrm{~cm}^{3} / \mathrm{g}\right)$} & \multirow{2}{*}{$\mathrm{D}_{\mathrm{p}}(\mathrm{nm})$} \\
\hline & Theoretical & IC & & & \\
\hline GAC & & & 273.2 & 0.162 & 10.9 \\
\hline $\mathrm{nFe} / \mathrm{GAC}$ & 20 & 18.60 & 69 & 0.041 & 30.95 \\
\hline
\end{tabular}

IC: wt\% determined using ion-chromatography (IC); $\mathrm{S}_{\mathrm{BET}}$ : BET surface area; $\mathrm{V}_{\mathrm{p}}$ : Total pore volumes were obtained at $\mathrm{P} / \mathrm{P}_{\mathrm{o}}=0.99$; $\mathrm{D}_{\mathrm{p}}$ : Average pore diameter calculated by BJH method.
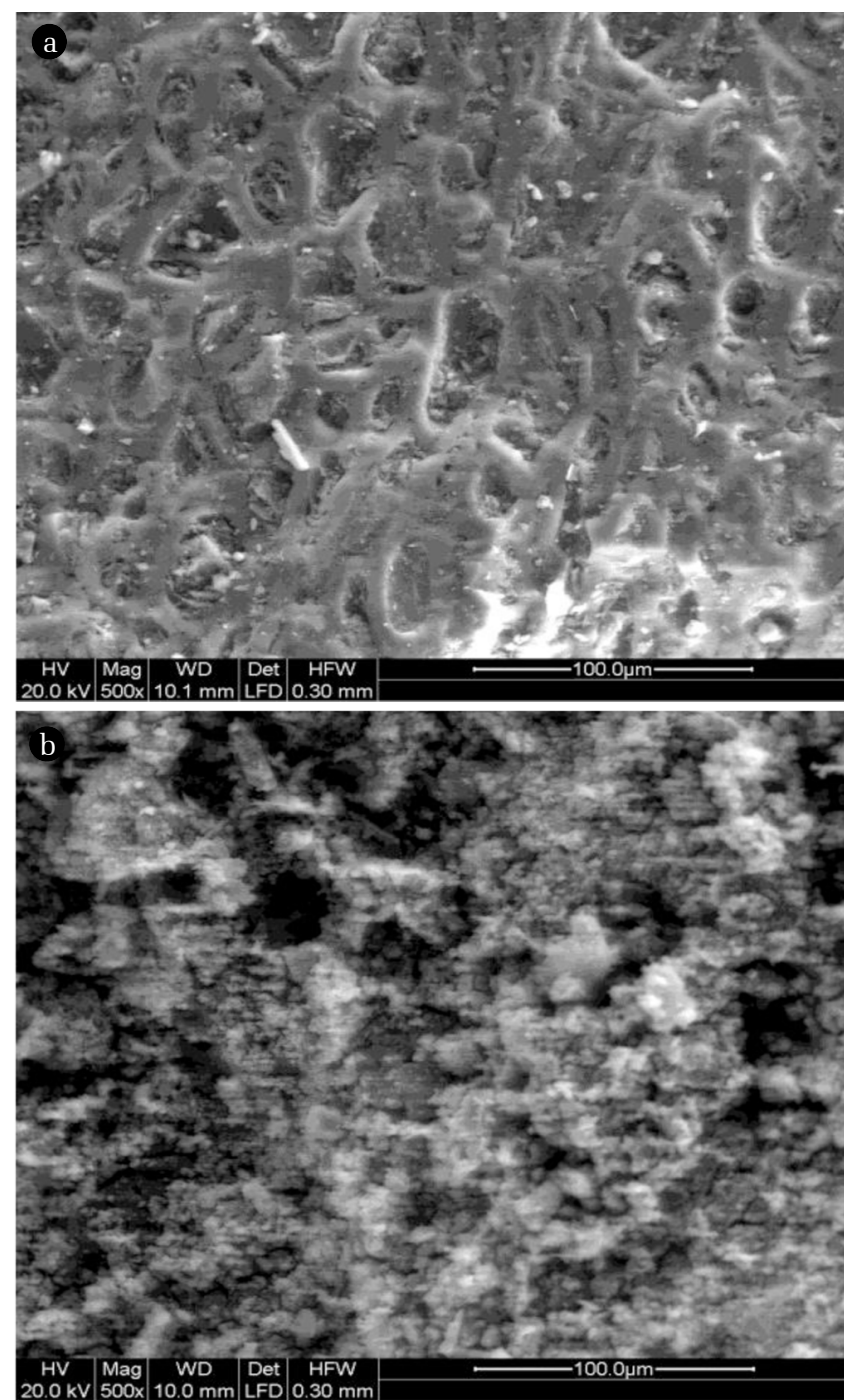

Fig. 2. SEM images of (a) GAC, (b) $20 \%-n \mathrm{Fe}^{0} / \mathrm{GAC}$.

that the adsorption branches of isotherm were horizontal and parallel to the desorption branches it indicates the possibility of the existence of slit pores in dominant form [19]. Passe-Coutrin et al. [20] reported that activated carbon exhibits type IV hysteresis due to the presence of ink-bottle type pore with slit type pore structure in the activated carbon framework. Table 3 depicted the textural properties such as surface area, pore volume and average pore diameter of the samples of bare GAC and $\mathrm{nFe}^{0} / \mathrm{GAC}$. Moreover,
$\mathrm{nFe}^{0}$ impregnated onto GAC decreased the corresponding BET surface area from $273 \mathrm{~m}^{2} / \mathrm{g}$ to $69 \mathrm{~m}^{2} / \mathrm{g}$. In addition, the pore volume also decreased from 0.162 to $0.041 \mathrm{~cm}^{3} / \mathrm{g}$. The above observation is due to the blocking of pores (growth of $\mathrm{nFe}^{0}$ ) of GAC by excess loading of $\mathrm{nFe}^{0}[18,10]$.

Fig. 2 indicates the SEM image of GAC and $20 \% \mathrm{nFe}^{0} / \mathrm{GAC}$. The GAC images illustrate the porous nature and $20 \% \mathrm{nFe} / \mathrm{GAC}$ shows the formation of the uniform film on the surface of GAC. At very high loading (above 10\% weight) of active species (Iron) onto the porous support, the channels of porous material (GAC) get saturated with multilayer adsorption. Further, indicates the blocking of the channel which leads to the decrease in the surface area of the material. SEM image of $20 \% \mathrm{nFe}^{0} / \mathrm{GAC}$ is good agreement with the results of the BET surface area. For bare GAC has a surface area of $273 \mathrm{~m}^{2} / \mathrm{g}$ and after loading of active metal the surface area was decreased drastically to $69 \mathrm{~m}^{2} / \mathrm{g}$ due to blocking of pores with an excess quantity of active metal. Subbaramaiah et al. [6] studied the different percentage (5\%, $10 \%$ and $20 \%$ ) of active metal $(\mathrm{Cu})$ loading on SBA-15. At higher loading $(20 \% \mathrm{Cu} / \mathrm{SBA}-15)$ of active metal the surface area was decreased drastically from $650 \mathrm{~m}^{2} / \mathrm{g}$ to $313 \mathrm{~m}^{2} / \mathrm{g}$ because of blocking of pores. The morphology of $\mathrm{nFe}^{0} / \mathrm{GAC}$ has been found as a uniform film, which is similar to trends reported in other studies [21].

\subsection{Fitting Model and Analysis of Variance (ANOVA)}

The optimum process parameters such as initial $\mathrm{pH}$, catalyst dose, oxidant dose, the concentration of Py and Qn determined by BBD analysis. TOC removal and Fe leaching were used as response value to perform the analysis using RSM. The BBD design matrix for real values together with experimental and predicted values in terms of percentage removal of TOC and total Fe leaching are tabulated in Table 2. The results of BBD were used to perform the analysis of variance (ANOVA) using Expert Design software (trial version). A polynomial regression model was found to be the best suitable fit between the response variables and input process variables. The calculated regression model was a function of initial $\mathrm{pH}$ (A), hydrogen peroxide dose (B), catalyst dose (C), Py conc. (D), and Qn conc. (E). The best fit of the responses with coded factors are given below (Eq. (3) and (4)).

$$
\begin{aligned}
Y_{\text {TOC }}= & 56.50-8.2 A+6.03 B+7.22 C-5.57 D-7.02 E-6.64 A^{2} \\
& +2.03 B^{2}-1.47 C^{2}+10.26 D^{2}+8.02 E^{2}+5.92 A B+1.35 A C \\
& +6.36 A D+5.76 A E+0.94 B C+3.92 B D+12.60 B E-4.71 C D \\
& -0.34 C E+10.74 D E
\end{aligned}
$$




$$
\begin{aligned}
Y_{\text {Tot.Feleaching }}= & 1.17-0.16 A-0.044 B+0.21 C-0.01 D+0.17 E \\
& -0.23 A^{2}-0.26 B^{2}-0.23 C^{2}-0.46 D^{2}-0.11 E^{2} \\
& -0.41 A B+0.12 A C-0.28 A D-0.75 A E-0.049 B C \\
& -0.034 B D-0.12 B E-0.078 C D-0.35 C E-0.19 D E
\end{aligned}
$$

For each response, the adequacy of the model was justified with the analysis of variance (ANOVA) and the results are shown in Table S1(a) and (b), that the liability of the model was extremely significant. In the obtained regression models (Eq. (3) and (4)), the positive sign indicates the positive effect on the removal of TOC and total iron leaching, respectively. Similarly, negative coefficients signify the negative effect on the removal of TOC, and total iron leaching, respectively. The "Prob $>\mathrm{F}^{\prime \prime}$ is the probability that all the variation in the results are due to random error [22]. When "Prob > F" is less than 0.05, terms in the model have a significant effect on response listed in the Table S1(a) and (b). The significant model terms for TOC removal are $\mathrm{A}, \mathrm{B}, \mathrm{C}, \mathrm{E}, \mathrm{AB}, \mathrm{AC}, \mathrm{AD}, \mathrm{AE}$, $\mathrm{BC}, \mathrm{BD}, \mathrm{BE}, \mathrm{CD}, \mathrm{DE}, \mathrm{A}^{2}, \mathrm{~B}^{2}, \mathrm{C}^{2}, \mathrm{D}^{2}$ and $\mathrm{E}^{2}$. While, for total Fe leaching $\mathrm{A}, \mathrm{B}, \mathrm{C}, \mathrm{E}, \mathrm{AB}, \mathrm{AC}, \mathrm{AD}, \mathrm{AE}, \mathrm{BE}, \mathrm{CD}, \mathrm{CE}, \mathrm{DE}, \mathrm{A}^{2}, \mathrm{~B}^{2}, \mathrm{C}^{2}, \mathrm{D}^{2}$ and $\mathrm{E}^{2}$ are significant model terms. In addition, values of "Prob $>\mathrm{F}^{\prime \prime}$ greater than 0.1 specifies the insignificant model terms. The F- values for TOC and total Fe leaching are 1594.82 and 75.89, which suggested that the terms in the model have a significant effect on response. The F- values could be larger due to noise. And the desirable adequate precision is always greater than four, and for TOC and total iron leaching it was found to be 198.018 and 38.75, respectively. Further affirmed the convenience of the model to navigate the design space. The $\mathrm{P}$ value represents the lack of fit which corresponds to 1.00 entail the insignificant as compared to the net error. Further regression model provides better knowledge in connection with five factors and their response [23].

\subsection{Adequacy of the Test Model}

The diagnostic plot of actual versus predicted and normal probability plots are acquired from the adequacy of the model. These plots provide information between actual values and predicted values and also assist to evaluate the model fitness. From Fig. 3(b), the data points

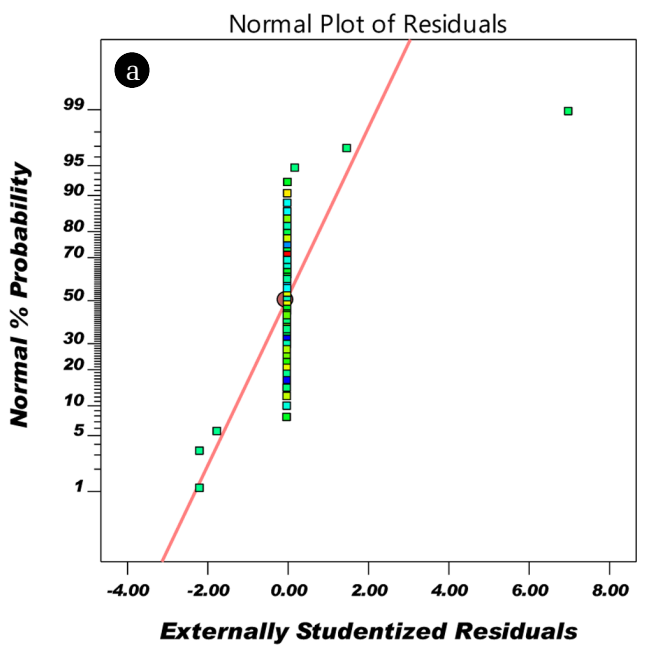

between the actual value and predicted values, located closed to a diametrical line attributed to good concurrence with the fitted model [24]. In addition, the prediction of the adequacy of the model can be determined by residual normality, Fig. 3(a) depicted the studentized residuals of a normal probability plot. No deviation was observed between observed residual and expected values [23, 25]. Two models namely i) the sequential model sum of squares, and ii) sequential model summary statistics were assessed to evaluate the adequacy of the test models (Table S2). Based on Table S2, the quadratic model is the best fit among other models. Furthermore, the $\mathrm{R}^{2}$ and adjusted $R^{2}$ are closely related to each other which indicate a good correlation between the experimental and predicted values.

\subsection{Effect of Parameters on Response}

Simultaneous mineralization of Py and Qn by catalytic wet peroxidation process was carried out in a batch study in the presence of the heterogeneous catalyst ( $\mathrm{nFe}^{0} / \mathrm{GAC}$ ) using hydrogen peroxide as oxidant. In this process nanoscale zero-valent iron utilizes the available dissolved oxygen converted into hydrogen peroxide and $\mathrm{Fe}^{2+}$. The formed hydrogen peroxide, $\mathrm{Fe}^{2+}$, and externally added hydrogen peroxide lead to generation of hydroxyl radical and $\mathrm{Fe}^{3+}$, the generated hydroxyl radical has high oxidation potential and is responsible for the oxidation/mineralization of organic compound (NHCs) (R) present in the reaction mixture. The generated hydroxyl radicals are non-selectively degrade the organic compound (NHCs) (R) into intermediate compounds $\left(\mathrm{R}^{*}\right)$ and further oxidation of these intermediate compounds $\left(\mathrm{R}^{\star}\right)$ into simple harmless compounds which are illustrated in below reaction scheme (Eqs. (5), (6) and (7)) $[9,18]$.

$$
\begin{aligned}
& n F e^{0} / \mathrm{GAC}+\mathrm{O}_{2} \rightarrow \mathrm{Fe}^{2+} / \mathrm{GAC}+\mathrm{H}_{2} \mathrm{O}_{2} \\
& \mathrm{Fe}^{2+} / \mathrm{GAC}+\mathrm{H}_{2} \mathrm{O}_{2} \rightarrow \mathrm{Fe}^{3+} / \mathrm{GAC}+{ }^{\circ} \mathrm{OH}+\mathrm{OH}^{-} \\
& \cdot \mathrm{OH}+\mathrm{R} \rightarrow \mathrm{R}^{*}+{ }^{\cdot} \mathrm{OH} \rightarrow \mathrm{CO}_{2}+\mathrm{H}_{2} \mathrm{O}+\mathrm{N}_{2} \mathrm{OrNO}_{x}
\end{aligned}
$$

Where: R: Organic compound (NHCs); R $^{*}$ Intermediate compounds

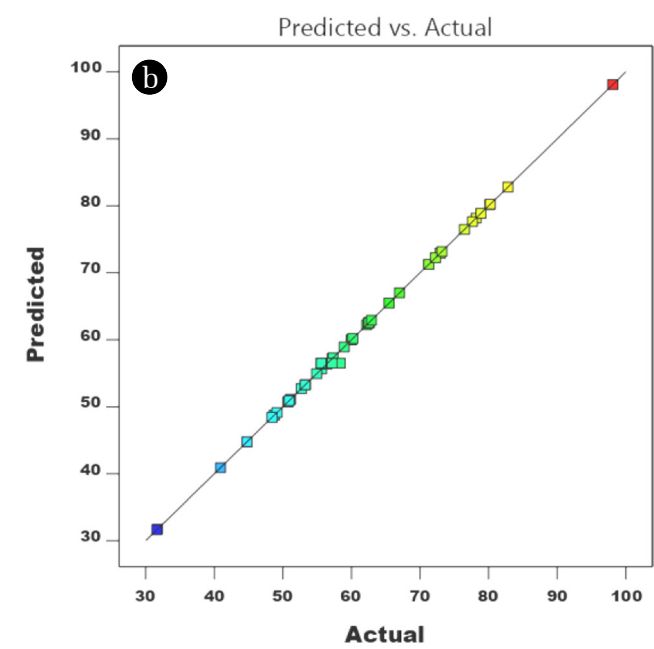

Fig. 3. (a) Normal probability plot of studentized residual (b) Predicted and Actual TOC removal. 
In order to gain the better understanding of the effects of independent variables and their interaction on TOC removal and total Fe leaching, 3D surface response plots and 2D contour plots were constructed based on the quadratic model. Moreover, 2D contour plots provide a straightforward assessment of the effects of experimental factors on the responses. In addition, an elliptical or saddle nature of the contour plots indicates the Interaction between the corresponding variables are significant [26]. Furthermore, each contour line designates the infinite number of arrangement of the selected process variables. The displayed plots reasonably interact with the process variables. All plots are nonlinear with selected process variables. Detailed effects of the process variable are discussed in the following section.

\subsubsection{Effect of $\mathrm{pH}$}

$\mathrm{pH}$ is one of the significant factor in CWPO process, which influences the performance of the oxidation process in the degradation of organic compounds. It plays an important role in controlling catalytic activity, stability of hydrogen peroxide and activity of iron species [27]. It can be seen in Fig. 4(a), (b), (c) and (d), that removal
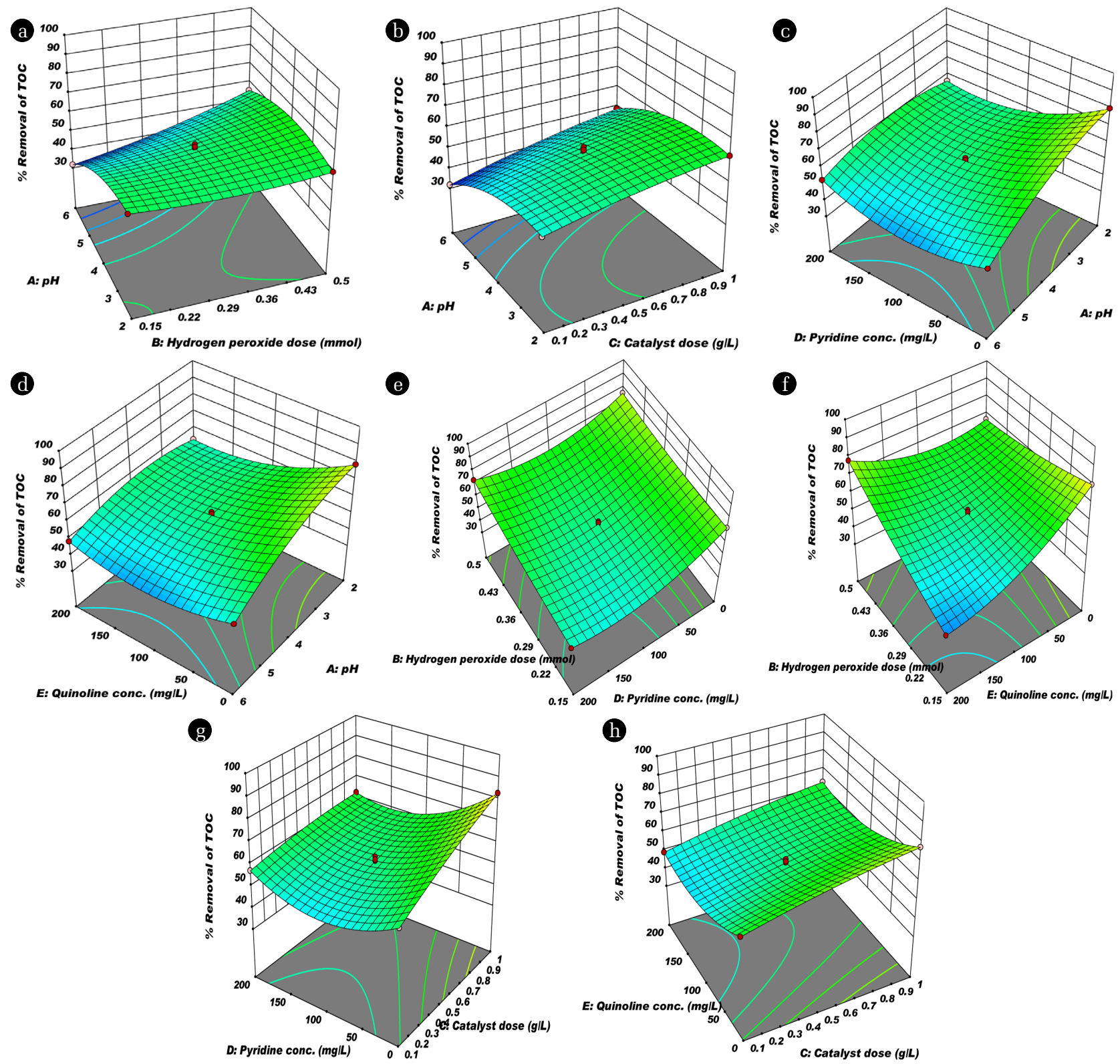

Fig. 4. \% Removal of TOC (In each effect other parameters kept at their center position such as dose $0.5 \mathrm{~g} / \mathrm{L} ; \mathrm{H}_{2} \mathrm{O}_{2} 0.3 \mathrm{mmol} ; \mathrm{pH} 4 ; \mathrm{Py}$ Conc. $100 \mathrm{mg} / \mathrm{L}$; and Qn Conc. $100 \mathrm{mg} / \mathrm{L}$ ). (a) Initial $\mathrm{pH}$ and $\mathrm{H}_{2} \mathrm{O}_{2}$ dose, (b) Initial pH and Catalyst dose, (c) Py conc. and Initial pH, (d) Qn conc. and Initial pH, (e) $\mathrm{H}_{2} \mathrm{O}_{2}$ dose and Py conc., (f) $\mathrm{H}_{2} \mathrm{O}_{2}$ dose and Qn conc., (g) Py conc. and Catalyst dose, (h) Qn Conc. and Catalyst dose. 

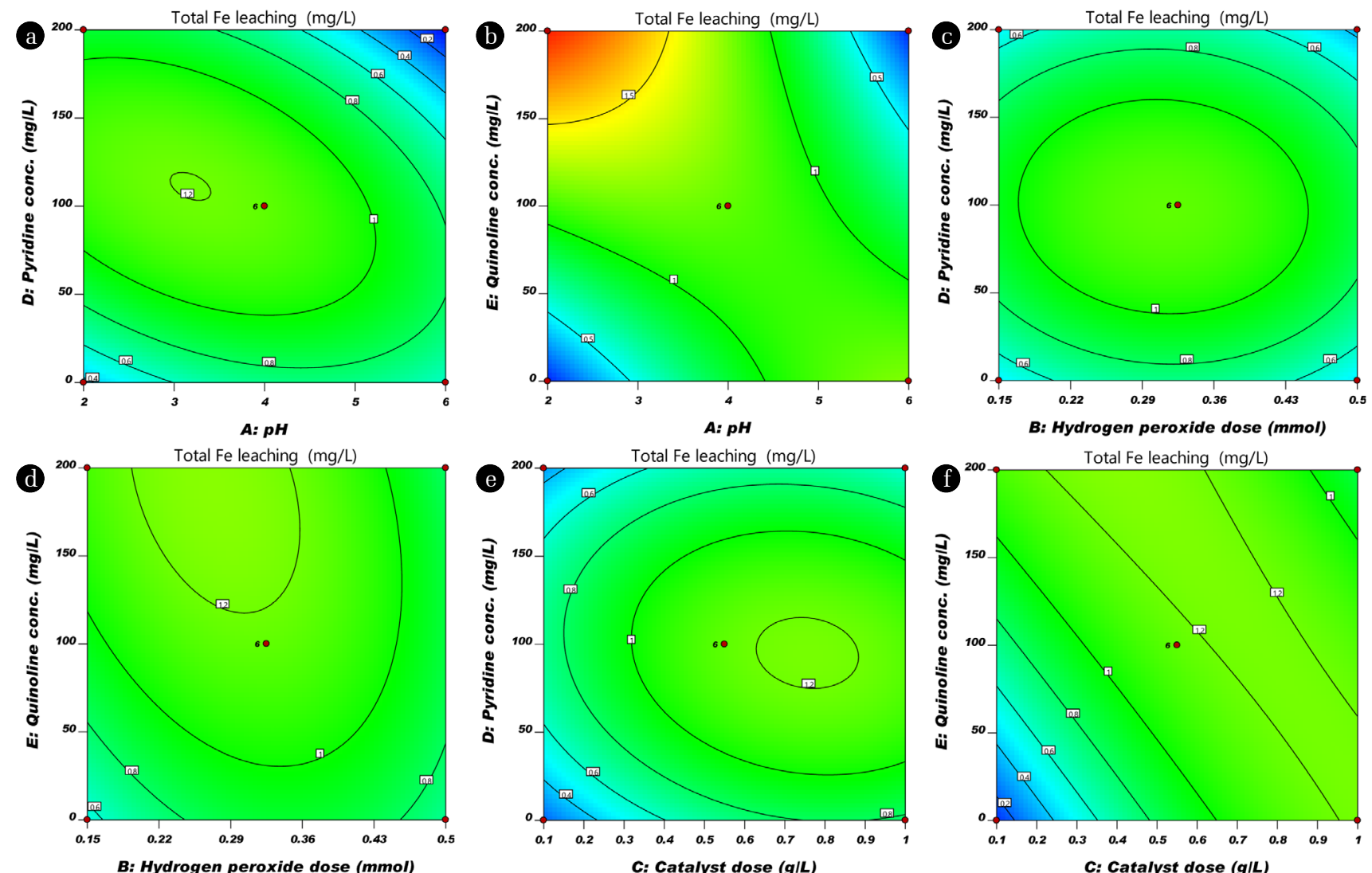

Fig. 5. Total Fe leaching (In each effect other parameters kept at their center position such as dose $0.5 \mathrm{~g} / \mathrm{L} ; \mathrm{H}_{2} \mathrm{O}_{2} \quad 0.3 \mathrm{mmol} ; \mathrm{pH} 4 ; \mathrm{Py}$ Conc. $100 \mathrm{mg} / \mathrm{L}$; and Qn Conc. $100 \mathrm{mg} / \mathrm{L}$ ). (a) Py conc. and Initial pH, (b) Qn conc. and Initial pH, (c) Py conc. and $\mathrm{H}_{2} \mathrm{O}_{2}$ dose, (d) Qn conc. and $\mathrm{H}_{2} \mathrm{O}_{2}$ dose, (e) Py conc. and Catalyst dose, (f) Qn conc. and Catalyst dose.

of TOC increased when initial $\mathrm{pH}$ value increased from 2-4. The low removal of TOC below $\mathrm{pH} 3$ might be attributed to scavenging of hydroxyl radical with $\mathrm{H}^{+}$ions ( ${ }^{\circ} \mathrm{OH}+\mathrm{H}^{+} \rightarrow \mathrm{H}_{2} \mathrm{O}$ ) [9]. Maximum removal was observed around 4 initial $\mathrm{pH}$. While the initial $\mathrm{pH}$ increased from 4 to 6 the TOC removal rate was declined, which limits the production of hydroxyl radical on the surface of $\mathrm{nFe}^{0}$. Thus, lead to slow mineralization of organic compound (low TOC removal) [28]. The total Fe leaching of NHCs are depicted in Fig. 5. Fig. 5(a) of $\mathrm{pH}$ vs $\mathrm{Py}$ concentration contour plots on total $\mathrm{Fe}$ leaching shows an elliptical nature attributed to the significant interaction between the $\mathrm{pH}$ and $\mathrm{Py}$ concentration [26]. The total Fe leaching was within the range of permissible limit $(3 \mathrm{mg} / \mathrm{L})$ of wastewater discharge in India (CPCB 2001). Therefore, it is concluded that from the above study that the initial $\mathrm{pH}$ value greater than 4 , more hydrogen peroxide was decomposed without appreciable improvement of CWPO.

\subsubsection{Effect of catalyst dose}

The influence of the catalyst dose in the range of $0.1-1 \mathrm{~g} / \mathrm{L}$ was studied with respect to the removal of TOC. Fig. 4(b), (g), and (h) demonstrate the removal of TOC with catalyst dose. Mineralization increased with increment of catalyst dose. At higher catalyst dose, the greater number of active sites are available which are responsible to generate the more hydrogen peroxide by oxidation of $\mathrm{Fe}^{0}$ with available dissolved oxygen into $\mathrm{Fe}^{2+}$ (Eq. (5)). The formed oxidized species $\left(\mathrm{Fe}^{2+}\right)$ again utilizes the available hydrogen peroxide (generated and external added hydrogen peroxide) convert into $\mathrm{Fe}^{3+}$ and hydroxyl radical (Eq. (6)). Further, presence of more number of active species $\left(\mathrm{Fe}^{0}\right.$ or $\left.\mathrm{Fe}^{2+}\right)$ leads to the generation of more quantity of hydroxyl radical. This hydroxyl radical oxidized the organic molecules into various organic acids and further these organic acids utilize the hydroxyl radical to convert into harmless compounds. Gosu et al. [18] proposed the degradation mechanism of Qn with hydroxyl radical using TOC removal. Fig. 5(f) shows the contour plot of catalyst dose vs Qn on iron leaching; it was observed that almost parallel line in 2D contour plot which represents the non interactive influence of these two parameters on iron leaching. Similarly catalyst dose vs Py on iron leaching in contour plots shows the elliptical nature due to the significant interaction between the catalyst dose and Py concentration on Fe leaching (Fig. 5(e)). Even though, the catalyst dose of $\mathrm{nFe}^{0} / \mathrm{GAC}$ is increased in the range of $0.1-1 \mathrm{~g} / \mathrm{L}$, the total Fe leaching is within the range of permissible limit of wastewater discharge in India shown in Fig. 5 [29].

\subsubsection{Effect of hydrogen peroxide}

CWPO effectiveness mainly depends on the generation efficiency of oxidizing species such as hydroxyl radicals. Moreover, powerful 
radicals are produced by the decomposition of hydrogen peroxide in the presence of $n F^{0}$ catalyst. Fig. 4(a), (b), (e), and (f) depicted the impact of hydrogen peroxide concentration on TOC removal. Furthermore, the TOC removal was improved as increase in the dose of hydrogen peroxide significantly because increase the number of hydroxyl radical generation for the mineralization of organic compound which leads the high TOC removal (Eq. (7)). Fig. 5(c) depicted the circular pattern of the lines in the contour plot suggested for the weak interactive influence of hydrogen peroxide vs. Py on total iron leaching. Further, indicate that Py mineralization path generates less number of acid intermediates that lead to low interaction of iron leaching into the aqueous solution. The elliptical view of lines in 2D contour plot (Fig. 5(d)) suggested for the significant interactive influence of hydrogen peroxide dose vs Qn concentration on total Fe leaching. This may depend on degradation path and type of intermediate compounds formation, if more number of intermediate acids are formed that leads to more leaching of iron into solution.

\subsubsection{Effect of initial concentration}

Organic compound concentration is an important operational factor as it influences the removal of TOC in the CWPO process. Fig. 4(c), (d), (e), (f), (g) and (h) describes the declining trend of TOC with an increase in the initial concentration of NHCs (Py and Qn concentration). As the more NHCs molecules may be adsorbed on active site of $\mathrm{nFe}^{0} / \mathrm{GAC}$, which become unavailable for hydrogen peroxide molecules to reach the active site for the generation of hydroxyl radical, and thus leads to lower degradation. The same observation was observed in interaction plot of Fig. 4(e), and (f), as TOC removal rate increased with an increase in the initial concentration of NHCs along with increase in hydrogen peroxide dose with their respective experimental ranges. Among these, Quinoline degradation shown the better efficiency when compare with pyridine. Higher rate of quinoline degradation can be attributed due to more electron density of nitrogen in quinoline molecule with respect to pyridine molecule. Longuet-Higgins and Coulson [30] calculated the $\Pi$-electron density of pyridine and quinoline, it was found that quinoline have higher П-electron density which leads the greater the ease and rate of cationoid substitution at that position.

Effect of Initial concentration of organic molecules on total $\mathrm{Fe}$ leaching was observed in 2D contour plot, Fig. 5(a), (c), (d), and (e) shows catalyst dose and hydrogen peroxide concentration interaction with pyridine concentration depicted circular pattern of the lines, which attributed to the weak interaction of two variables on total Fe leaching [26]. Furthermore, for Qn concentration with initial $\mathrm{pH}$ and catalyst dose shows an almost parallel line in 2D contour plot (Fig. 5(b) and (c)), it indicates that almost no interactive influence of these two variables on the total Fe leaching. It was found that, all treated sample Fe leaching was below $2 \mathrm{mg} / \mathrm{L}$. The permissible limit of $\mathrm{Fe}$ in wastewater discharge is $3 \mathrm{mg} / \mathrm{L}$ (CPCB, Environment (protection) rules 1986). According to the present study, further Fe treatment is not necessary. In case if it is exceeded any other process by active metal leaching beyond permissible limit one can recommend simple adsorption process as simple post-treatment process.

\subsection{Optimization Using Desirability Function}

In the present study, multi-response optimization by desirability function approach was used for the optimization of the process variables by Derringer's desirability function. As shown in Table S3, the desired goal was selected for each process variable and response. The each goal was changed with respect to our desired goals. Further, 10 optimum points are generated through numerical simulation. Among those, the best optimum conditions are initial solution $\mathrm{pH}$ of 3.5 , hydrogen peroxide dose of $0.34 \mathrm{mmol}$, catalyst dose of $0.55 \mathrm{~g} / \mathrm{L}$, Py concentration of $200 \mathrm{mg} / \mathrm{L}$ and Qn concentration of $200 \mathrm{mg} / \mathrm{L}$, at which TOC removal of $84 \%$ has suggested with the desirability of 0.7 . The experiments are conducted in duplicate to validate the model (Table S4). The predicted values nearly close to experimental values obtained from optimization analysis (Table S4).

\section{Conclusions}

Catalytic wet peroxidation process was used for the simultaneous oxidation of Py and Qn containing wastewater in the presence of nanoscale zero-valent iron with the help of Box-Behnken design. The five variables including initial $\mathrm{pH}$, oxidant dose, catalyst dose and concentration of Py and Qn were evaluated based on TOC removal. From BBD, concluded that all five parameters are effective parameters on TOC removal. The F- values of TOC and total iron leaching are 1,594.82 and 75.89, which suggested that the terms in the model have a significant effect on response. $\sim 84 \%$ TOC removal was observed in binary compounds oxidation at initial solution $\mathrm{pH}$ of 3.5 , catalyst dose of $0.55(\mathrm{~g} / \mathrm{L})$, hydrogen peroxide dose of $0.34 \mathrm{mmol}$, Py concentration of $200 \mathrm{mg} / \mathrm{L}$ and Qn concentration of $200 \mathrm{mg} / \mathrm{L}$.

\section{References}

1. Marella A, Tanwar OP, Saha R, et al. Quinoline: A versatile heterocyclic. Saudi Pharm. J. 2013;21:1-12.

2. Sims GK, O'Loughlin EJ, Crawford RL. Degradation of pyridines in the environment. Crit. Rev. Environ. Control. 1989;19:309-340.

3. Fetzner S. Bacterial degradation of pyridine, indole, quinoline, and their derivatives under different redox conditions. Appl. Microbiol. Biot. 1998;49:237-250.

4. Mensah K, Forster C. An examination of the effects of detergents on anaerobic digestion. Bioresour. Technol. 2003;90:133-138.

5. Barrabés N, Sá J. Catalytic nitrate removal from water, past, present and future perspectives. Appl. Catal. B Environ. 2011;104:1-5.

6. Subbaramaiah V, Srivastava VC, Mall ID. Optimization of reaction parameters and kinetic modeling of catalytic wet peroxidation of picoline by $\mathrm{Cu} / \mathrm{SBA}-15$. Ind. Eng. Chem. Res. 2013;52:9021-9029.

7. Mahdad F, Younesi H, Bahramifar N, Hadavifar M. Optimization of Fenton and photo-Fenton-based advanced oxidation processes for post-treatment of composting leachate of municipal solid waste by an activated sludge process. KSCE J. Civ. Eng. 2016;20:2177-2188.

8. Zrncevic S, Gomzi Z. CWPO: An environmental solution for pollutant removal from wastewater. Ind. Eng. Chem. Res. 
2005;44:6110-6114.

9. Subbaramaiah V, Srivastava VC, Mall ID. Catalytic activity of $\mathrm{Cu} / \mathrm{SBA}-15$ for peroxidation of pyridine bearing wastewater at atmospheric condition. AIChE J. 2013;59:2577-2586.

10. Subbaramaiah V, Srivastava VC, Mall ID. Catalytic wet peroxidation of pyridine bearing wastewater by cerium supported SBA-15. J. Hazard. Mater. 2013;248:355-363.

11. Kim DG, Hwang YH, Shin HS, Ko SO. Kinetics of nitrate adsorption and reduction by nano-scale zero valent iron (NZVI): Effect of ionic strength and initial pH. KSCE J. Civ. Eng. 2015;20: 175-187.

12. Gosu V, Gurjar BR, Surampalli RY, Zhang TC. Oxidative degradation of quinoline using nanoscale zero-valent iron supported by granular activated carbon. J. Environ. Eng. 2016;142: 04015047-11.

13. Üzüm C, Shahwan T, Eroğlu AE, Hallam KR, Scott TB, Lieberwirth I. Synthesis and characterization of kaolinite-supported zero-valent iron nanoparticles and their application for the removal of aqueous $\mathrm{Cu}^{2+}$ and $\mathrm{Co}^{2+}$ ions. Appl. Clay Sci. 2009;43:172-181.

14. Gosu V, Gurjar BR, Surampalli RY, Zhang TC. NFe $/$ GAC-mediated advanced catalytic per-oxidation for pharmaceutical wastewater treatment. J. Environ. Chem. Eng. 2014;2:1996-2004.

15. Simsek EB, Tuna AA, Beker U. A statistical approach for arsenic adsorption onto turkey clinoptilolite. Environ. Sci. Pollut. R. 2015;22:3249-3256

16. Anotai J, Thuptimdang P, Su CC, Lu MC. Degradation of o-toluidine by fluidized-bed Fenton process: statistical and kinetic study. Environ. Sci. Pollut. Res. 2012;19:169-176.

17. Rice EW, Baird RB, Eaton AD, Clesceri LS. Standard methods for the examination of water and wastewater. 22nd eds. Washington, DC: American Public Health Association; 2012. p.440-441.

18. Gosu V, Gurjar BR, Surampalli RY, Zhang TC. Treatment of pyridine-bearing wastewater by nano zero-valent iron supported on activated carbon derived from agricultural waste. Desalin. Water Treat. 2015;57:6250-6260.

19. Marsh H. Activated carbon compendium: A collection of papers from the journal carbon 1996-2000. 1st ed. Amsterdam: Elsevier;
2001. p. 220-221.

20. Passe-Coutrin N, Altenor S, Cossement D, Jean-Marius C, Gaspard S. Comparison of parameters calculated from the BET and Freundlich isotherms obtained by nitrogen adsorption on activated carbons: A new method for calculating the specific surface area. Micropor. Mesopor. Mater. 2008;111:517-522.

21. Xi Y, Megharaj M, Naidu R. Dispersion of zerovalent iron nanoparticles onto bentonites and use of these catalysts for orange II decolourisation. Appl. Clay Sci. 2011;53:716-722.

22. Segurola J, Allen NS, Edge M, Mahon AM. Design of eutectic photoinitiator blends for UV/visible curable acrylated printing inks and coatings. Prog. Org. Coat. 1999;37:23-37.

23. Ahmadi M, Ghanbari F. Optimizing COD removal from greywater by photoelectro-persulfate process using Box-Behnken design: Assessment of effluent quality and electrical energy consumption. Environ. Sci. Pollut. R. 2016;23:19350-19361.

24. Rezaei M, Rashidi F, Royaee SJ, Jafarikojour M. Performance evaluation of a continuous flow photocatalytic reactor for wastewater treatment. Environ. Sci. Pollut. R. 2014;21:12505-12517.

25. Moradi M, Ghanbari F, Manshouri M, Angali KA. Photocatalytic degradation of azo dye using nano-ZrO2/UV/persulfate: response surface modeling and optimization. Korean J. Chem. Eng. 2015;33:539-546.

26. Singh KP, Singh AK, Singh UV, Verma P. Optimizing removal of ibuprofen from water by magnetic nanocomposite using Box-Behnken design. Environ. Sci. Pollut. R. 2012;19:724-738.

27. Babuponnusami A, Muthukumar K. Removal of phenol by heterogeneous photo electro Fenton-like process using nano-zero valent iron. Sep. Purif. Technol. 2012;98:130-135.

28. Liu X, Wang F, Chen Z, Megharaj M, Naidu R. Heterogeneous Fenton oxidation of Direct Black $G$ in dye effluent using functional kaolin-supported nanoscale zero iron. Environ. Sci. Pollut. R. 2014;21:1936-1943.

29. CPCB, Pollution control acts, rules and notifications issued there under central pollution control board Govt. of India, 2001.

30. Longuet-Higgins HC, Coulso CA. A theoretical investigation of the distribution of electrons in some heterocyclic molecules containing nitrogen. Trans. Faraday Soc. 1947;43:87-94. 